\title{
The Hikikomori Phenomenon: Could Loneliness Be a Choice of Self-Restriction from Society?
}

\author{
Giulio Castelpietra1,2, Alessandra Nicotra², Diego De Leo ${ }^{3}$ \\ ${ }^{1}$ Primary Care Services Area, Central Health Directorate, Regione autonoma Friuli Venezia Giulia, Trieste, Italy \\ ${ }^{2}$ Department of Medicine (DAME), University of Udine, Udine, Italy \\ ${ }^{3}$ Australian Institute for Suicide Research and Prevention, Griffith University, Brisbane, Australia \\ Email: giulio.castelpietra@regione.fvg.it,d.deleo@griffith.edu.au
}

How to cite this paper: Castelpietra, G., Nicotra, A. and De Leo, D. (2021) The Hikikomori Phenomenon: Could Loneliness Be a Choice of Self-Restriction from Society? Open Journal of Psychiatry, 11, 47-62. https://doi.org/10.4236/ojpsych.2021.111005

Received: December 14, 2020

Accepted: January 25, 2021

Published: January 28, 2021

Copyright (c) 2021 by author(s) and Scientific Research Publishing Inc. This work is licensed under the Creative Commons Attribution International License (CC BY 4.0).

http://creativecommons.org/licenses/by/4.0/

\begin{abstract}
Originated in Japan, the hikikomori phenomenon-as a form of individual withdrawal-has progressively widespread and gained international attention, given its psycho-social and economic consequences. In fact, hikikomori appears today as a problem that cannot be confined solely to the field of mental health. Rather, it appears as a phenomenon that concerns many aspects of modern society, intersecting with medicine, sociology, anthropology, politics, economics, culture and education. In the context of the actual loneliness pandemic, hikikomori individuals present characteristics that are examined in this article, together with suggestions for its possible management.
\end{abstract}

\section{Keywords}

Hikikomori, Social Withdrawal, Loneliness, Internet Addiction, Mental Health

\section{Introduction}

The term hikikomori derives from the Japanese verbs hiki and komoru, which means "withdraw" and "come into" respectively [1]. T. Fujiya first used the term in 1985 to refer to retreat neurosis and student apathy. It later gained international attention with the 1998 publication of the book Shakaiteki Hikikomori: Owaranai Shishunki (Hikikomori: Adolescence without End) by the Japanese psychiatrist T. Saito. The phenomenon is widespread in Japan where it is estimated that upwards of one million people live reclusive lives as hikikomori.

The first guidelines about hikikomori were published by the Japanese Ministry of Health Labour and Welfare (MHLW) in 2003, defining hikikomori as a con- 
dition characterized by:

1) A lifestyle centred at home;

2) No interest or willingness to attend school or work;

3) Persistence of symptoms/behaviours beyond six months;

4) Schizophrenia, mental retardation or other mental disorders were to be excluded.

Among those with no interest or willingness to attend school or work, those who maintained personal relationships (e.g., friendships) were also to be excluded [2].

In 2010, the MHLW produced a more accurate description: "Hikikomori is a psycho-sociological phenomenon, one of its characteristic features being withdrawal from social activities and staying at home almost each day for more than half a year. This occurs among children, adolescents, and adults under 30 years old. Although hikikomori is defined as a non-psychotic state, it is better to think that patients of schizophrenia may be mixed into this grouping until they'll be receive the diagnosis of psychosis" [3].

In 2019, Kato et al. [4] provided an updated proposal of diagnostic criteria for hikikomori, which comprised the following criteria: 1) marked social isolation in one's home; 2) duration of continuous social isolation of at least 6 months; and 3) significant functional impairment or distress associated with the social isolation. This definition tried also to clarify the frequency of going outside home, and the duration of social withdrawal. However, the requirement for avoidance of social situations and relationships was removed, since hikikomori commonly report having few meaningful social relationships and little social interaction. Distress or functional impairment should be carefully evaluated, since subjective distress may not be present, albeit hikikomori people usually feel distressed as the duration of social withdrawal gets longer. Moreover, other psychiatric disorders as an exclusion criterion for hikikomori were removed, since hikikomori may undergo a variety of co-occurring psychiatric disorders as a contributor to psychopathology.

Various other forms of severe social withdrawal were described in Japan from the end of the 1970s [5]. For instance, the term taijin kyofusho describes young people who leave their jobs or schooling for long periods without having a diagnosis of depression or psychosis. Taijin kyofusho has been associated with social phobias or social anxiety disorders and has been classified in DSM-5 into the glossary of cultural concepts of distress [3].

However, unlike taijin kyofusho, hikikomori became a buzzword worldwide, in spite of a lack of consensus as to its definition. Indeed, the classification of hikikomori as a syndrome with clearly defined clinical features is still controversial [3]. This may be due to the fact that the term was originally limited to the Japanese context, and was strictly associated with sociocultural features of the country, including the increased income of Japanese families, more lenient parenting, and a general decline in desire and motivation among young adults [6]. Difficulties in labour market access and large economic changes are additional social 
factors acutely felt by younger generations. Moreover, the contrast between older traditions and a highly technologized society-a contrast that is particularly stark in contemporary Japan-may have contributed to a crisis of sociocultural values whose effects on adolescents could be particularly detrimental [5]. While hikikomori is often viewed as a negative phenomenon, it has been argued that in fact it could increase social connectedness and sense of identity through new means better suited to those who adopt them [7].

A growing body of research shows that hikikomori occurs not only in Japan, but also in neighbouring South Korea and China, as well as in countries as diverse as Oman, India, Spain, France, Italy, the United Kingdom, Canada, Australia and the United States [8]-[14]. Its occurrence may be due to the presence of social conditions similar to those in Japan, such as growing employment uncertainty and rapidly shifting social values [15]. The dramatic increase in social media use and online gaming, which permit indirect social interactions across vast distances, may have contributed to the global spread of the phenomenon [15] [16].

Alongside a growing scientific literature on hikikomori, the phenomenon is gaining a growing presence in common media. Its popular representations include a number of novels and graphic novels, and various blogs and YouTube channels dedicated to the topic. This prevalence in the media may contribute to a rise in cases, but has also contributed to increased awareness of the phenomenon [6].

With its complex causes and worldwide distribution, hikikomori appears today as a transversal phenomenon, so widespread that cannot be assimilated to a specific sociocultural context nor confined to the field of mental health [8]. Rather, it is a disorder that reaches deeply into all aspects of society, intersecting with anthropology, medicine, sociology, politics, economics, culture and education. Here, we briefly summarize and reflect upon the growing-but still scarce-body of literature that has described the complexity and extension of the hikikomori phenomenon. In this research note, we describe the magnitude of the phenomenon in relation to the few epidemiological data, discuss hikikomo$r i$ s sociological, familiar and clinical characteristics, and present possible therapeutic and rehabilitative management. Further, we shed a light on hikikomori phenomenon taking into account social withdrawal as one of its main aspects to analyse.

\section{Primary and Secondary Hikikomori}

Is hikikomori a mental disorder? Literature is starkly divided and the question remains unresolved. Although an inclusion of diagnostic criteria of hikikomori in international classifications of mental disorders, as DSM-5 or ICD-11, has recently been claimed, the claim remains controversial [17]. Several authors consider hikikomori an underground subculture for coping with societal issues such as bullying and discrimination [8]. Others identify hikikomori as a new form of depression [9]. As a result, some authors propose that hikikomori be differen- 
tiated between a "primary" form where comorbidities are absent and a "secondary" form where a diagnosis of psychiatric disorder should be added [6] [9] [10] [12] [18]. For instance, in a survey of hikikomori from Japan, half of those interviewed showed no comorbidities with psychiatric disorders, while the other half were shown to carry comorbid affective, anxiety, and substance use disorders [19].

However, since the identification of hikikomori is mainly based on social withdrawal, a major issue can be that individuals may withdraw for different reasons and in different ways, with a high level of heterogeneity across different cultures [15]. It has also been argued that primary hikikomori cannot be considered as s syndrome but only an idiom of distress; this definition presents a paradox in which a mental health disorder is created in order to categorize forms of social withdrawals that do not belong to the mental health field [3]. Other authors argue that social isolation is a symptom common to many mental disorders and therefore cannot be considered as a new diagnostic category [20]. It is clear, in fact, that the symptoms and behaviours that hikikomori often experience, such as social withdrawal, depressed mood, suicidal ideation or attempted suicide, could incline physicians to diagnose them with dysthymia, major depression or even schizophrenia or schizoid or avoidant personality disorders. It is also important to note that the correlation between hikikomori and suicidal behaviours is not as high as might be expected, leading to the hypothesis that many of the young people locked in their rooms may have found a way of life that is an acceptable alternative to suicide [21]. To date, it appears useful to differentiate between primary and secondary hikikomori, especially for the purpose of epidemiological data collection and the development of management strategies. However, it should be highlighted that the borders between primary and secondary hikikomori are not clearly defined. Although primary symptoms are a self-imposed state of extreme voluntary isolation, this can be interpreted both as a form of depression [21] or a form of defense against the transition from adolescence to adulthood [22].

\section{Epidemiology}

Although the number of case reports describing hikikomori has been increasing worldwide, epidemiological data on hikikomori using large samples is still scarce. This scarcity is the result of several factors. First, as described above, a clear definition of hikikomori is still lacking and there is no consensus on diagnostic criteria across different studies [10] [23]. Secondly, the aforementioned debate over whether hikikomori should be diagnosed in the presence of psychiatric comorbidity may influence data retrieving. Thirdly, there is a lack of validated tools to assess hikikomori across different countries and environments. While several established tools are available-including the "Hikikomori Behaviour Checklist" [24], the "Adaptive Behaviours Scale for Hikikomori (ABS-H)", addressed to parents of socially withdrawn children [25], and the "NEET/Hikikomori Risk Scale" that addresses occupational withdrawal and consequent marginaliza- 
tion from society [26] - these tools are in Japanese language and mainly intended for the Japanese context. The "25-item Hikikomori Questionnaire" (HQ-25) is a self-report instrument recently developed with the aim of investigating hikikomori behaviours across different cultures [27]. Although data at the population level are still lacking, hikikomori-like cases have been reported in a many other countries such as South Korea, Hong Kong, Mainland China, India, Spain, Italy, France, United States, and Brazil [28], as shown in Table 1.

Table 1. Hikikomori syndrome across different countries.

\begin{tabular}{|c|c|c|c|c|c|}
\hline Title of study & Year & Type of study & Country & Sample (N) & Findings \\
\hline $\begin{array}{l}\text { Hikikomori in Spain: } \\
\text { A descriptive study [12] }\end{array}$ & 2015 & Descriptive & Spain & $\mathrm{N}=164$ & $\begin{array}{l}\text { Hikikomori were predominantly young male, with the } \\
\text { mean age at onset of hikikomori of } 40 \text { years old and a } \\
\text { mean socially withdrawn period of } 3 \text { years. Only three } \\
\text { people had no symptoms suggestive of mental disorder. } \\
\text { Psychotic and anxiety were the most common comorbid } \\
\text { disorders }\end{array}$ \\
\hline $\begin{array}{l}\text { Home visitation program for } \\
\text { detecting, evaluating, and } \\
\text { treating socially withdrawn } \\
\text { youth in Korea [14] }\end{array}$ & 2013 & Case control & Korea & $\begin{array}{c}\mathrm{N}=41 \mathrm{vs} \\
\mathrm{N}=248\end{array}$ & $\begin{array}{l}\text { Over } 50 \% \text { of hikikomori cases had either passive or } \\
\text { indifferent relationships with peers and parents in early } \\
\text { life, which may correlate with the temperamental } \\
\text { outcome of their present status. Also, } 56 \% \text { of had } \\
\text { experienced school bullying, and most participants had } \\
\text { hints of one or more psychiatric diseases }\end{array}$ \\
\hline
\end{tabular}

A 12-month study of the hikikomori syndrome of social withdrawal: Clinical characterization and different subtypes proposal [20]

Identification of the hikikomori syndrome of social withdrawal: psychosocial features and treatment preferences in four countries [23]

Hikikomori: The Japanese Cabinet Office's 2016 Survey of Acute Social Withdrawal [29]

Lifetime prevalence, psychiatric comorbidity and demographic correlates of "hikikomor" in a community population in Japan [19]
Six major diagnostic groups: affective, anxiety, psychotic, drug use, personality and other Axis I disorders. Only one case corresponded to primary hikikomori. Social isolation was more frequent in men. The anxiety-affective group had lower clinical severity but worse evolution. Subjects undergoing intensive treatment had a higher medical follow up rate and better social networks at 12 months.

Participants had high levels of loneliness (UCLA Loneliness Scale $\mathrm{M}=55.4, \mathrm{SD}=10.5)$, limited social networks (LSNS-6 $\mathrm{M}=9.7, \mathrm{SD}=5.5$ ) and moderate functional impairment (SDS $M=16.5, \mathrm{SD}=7.9$ ). Of them 28 (78\%) desired treatment for their social withdrawal, with a significantly higher preference for psychotherapy over pharmacotherapy, in-person over telepsychiatry treatment and mental health specialists over primary care providers.

It estimated 541,000 cases of hikikomori, with prevalence 2017 Survey Japan $\quad \mathrm{N}=5000$ population of $1.6 \%$. $60 \%$ males, half between 20 to 29 years old, $70 \%$ unemployed

A total of $1.2 \%$ had experienced hikikomori in their lifetime. Among them, $54.5 \%$ had also experienced a psychiatric (mood, anxiety, impulse control, or substance-related) disorder in their lifetime. Respondents who experienced "hikikomori" had a 6.1 times higher risk of mood disorder. Among respondents, $0.5 \%$ currently had at least one child who had experienced "hikikomori". While psychiatric disorders were often comorbid with "hikikomori", half of the cases seem to be primary hikikomori without a comorbid psychiatric disorder. 
To confirm or to Maintain Self-Consistency? Hikikomori From Seeking Harmony [32] Risk in Japan and the Deviation

2014

Cohort

Japan

$\mathrm{N}=195$

The prevalence and correlates of severe social withdrawal (hikikomori) in Hong Kong: A cross-sectional telephone-based survey study [31]
Partecipants were divided into high risk vs. low risk of becoming hikikomori. High risk students shared similar social perceptions about pervasive values in their society as low risk students, thereby ruling out deficits in social perception as being associated with hikikomori risk. Instead, high risk students were not motivated to conform to others' behaviors relative to low-risk students and this difference was fully mediated by differences in preferred levels of harmony-seeking. Furthermore, high risk students scored lower on both local identity and global identity relative to low-risk students, thereby reifying their marginalized identity in Japanese society.

The prevalence rates of more than 6 months, less than 6 months and self-perceived non-problematic social withdrawal were $1.9 \%, 2.5 \%$ and $2.6 \%$, respectively. In terms of the correlates, the internationally and locally

2019 Cross sectional China $\quad \mathrm{N}=1010$ defined socially withdrawn youths are similar, while the self-perceived non-problematic group is comparable to the comparison group. The study finds that the prevalence of severe social withdrawal in Hong Kong is comparable to that in Japan.

In Japan, a survey on acute social withdrawal was performed in December 2015 on 5000 individuals aged 15 to 39 years old: it estimated 541,000 cases of hikikomori, with a population prevalence of $1.6 \%$ [29]. Of the hikikomori cases identified, $60 \%$ were males, half of the individuals were aged between 20 to 29 years, and almost 70\% were unemployed. Two Japan's Cabinet Office surveys in $2016 / 2019$ exposed that the estimated number of persons socially isolated for more than six months between 15 years old to 65 years old was 1,150,000. In Japan, epidemiological research conducted on general population samples has shown that the prevalence of the hikikomori can range from approximately $0.87 \%$ to $1.2 \%$ [30].

In Hong Kong, a telephone-based survey of social withdrawal behaviours involved 1010 individuals aged 12 - 29 years. The estimated prevalence of $1.9 \%$ was consistent with that of Japan [31]. The few epidemiological reports available from other countries are based on small samples. For example, a South Korean study suggested that $2.3 \%$ of high school students experience social withdrawal, but this study was based on only 41 individuals. An observational study from Barcelona, Spain, evaluated 164 cases of hikikomori referred to a Crisis Resolution Home Treatment program because of social isolation, but found only three people with primary hikikomori while the vast majority were diagnosed with psychotic and anxiety disorders [12]. Studies to date indicate that common contributing factors for hikikomori include male gender, an age younger than 30 years and a history of rejection from school and/or work. Comorbid psychiatric illnesses are very likely, especially in western countries [20]. High levels of loneliness and a lack of support from social networks have also been found across countries [23]. A possible reason that may explain these variations in prevalence 
may depend on differences in the inclusion criteria and in assessment instruments. In treatment-seeking population or in patients with psychiatric disorder, the prevalence can vary from $12.6 \%$ up to $63.1 \%$ [30].

\section{Sociological Theories}

Norasakunkkit and Uchidar believe that a relevant number (if not the greatest) of individuals identifiable as hikikomori are not carriers of a psychiatric disorder (including those on the autism spectrum) but are instead expressing their refusal to conform and accept existing social values and behaviors. Their social withdrawal would therefore represent a form of social avoidance and protest [32].

In Shutting out the Sun: How Japan Created its Own Lost Generation-one of the best-known books on hikikomori-author Michael Zielenziger claims that "to survive in Japan, you have to kill off your own original voice" [33]. Zielenziger's statement refers to the necessity of killing off individual differences andfeelings of dissatisfaction in order to conform to strict mainstream rules. Since in Japanese society work achievements depend on loyalty and accumulation of years of service, university and high school students-the age group of the majority of hikikomori-find the prospect of entering the labour market as daunting and distressing [21]. Zielenziger suggests that non-conforming young people create their own freedom at home by becoming hikikomori. He thus interprets hikikomori as a coping strategy to survive a stressful and high-pressure society in which youths fall at the bottom of the social pyramid (Table 2).

An understanding of the concept of amae can also help explain the general acceptance of hikikomori in Japanese society [5] [9]. Amae defines an excessive dependence of the child on his/her mother. After the Second World War, Japan underwent a change in the family structure, in which the traditional family was replaced by the nuclear family model common to Western countries, in which the father spends his days in wage labour while the mother is the first and most significant person responsible for raising children. The literature reports several cases of hikikomori characterized by an overprotective and intrusive mother who tends to manage the lives of her children and overcharge them with ideals and expectations. This type of relationship may also explain reported episodes of aggressiveness of hikikomori towards their mothers [5].

The topic of correlations between family relationships and hikikomori has

Table 2. Main etiological factors and hypothesis for development of hikikomori social withdrawal.

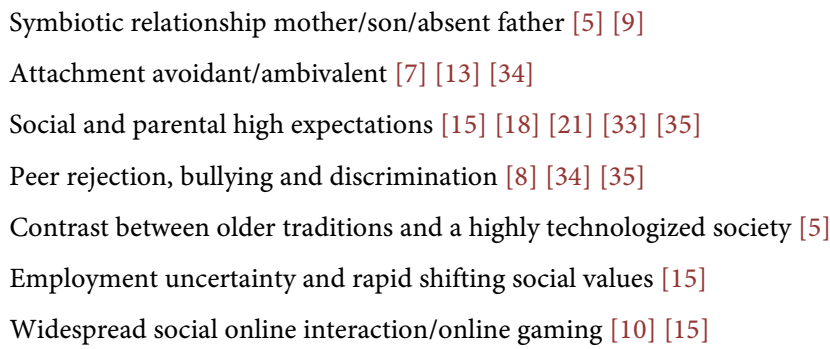


been further analysed in order to describe the link between social withdrawal in youth and avoidant or ambivalent attachment [7] [34] (Table 2). Avoidant individuals express social withdrawal as a behaviour to cope with nonresponsive attachment figures and attachment traumas [13], while ambivalent individuals are more likely to look for help by their attachment figures, albeit they are not always respondent, contributing to frustration and insecurity. It has been argued that cultural factors may amplify avoidant behaviour. For instance, in Japan youths may experience greater difficulty in dealing with the psychological impact of peer rejection. The combined effects of high social expectations and peer rejection can lead to social withdrawal [34] (Table 2). Suwa and Suzuki have described the development of a false identity of young hikikomori, characterized by five features: high parental expectations, an idealized self that fits others' desires rather than one's own, the need to conform to an image imposed by society, an episode of "defeat without a struggle" and the need to avoid further situations of this kind in order to protect the ideal self [18].

\section{Clinical Characteristics}

As summarized in Table 2, it seems plausible to consider hikikomori as an interaction of biopsychosocial, cultural and environmental factors, including parental and school determinants [35]. Clinical characteristics such as social withdrawal have been reported throughout the literature on hikikomori. Many of the symptoms are common to several mental conditions such as social deterioration, loss of motivation, dysphoric mood, anxiety symptoms and avoid social situations, making social anxiety disorder, sleep disturbance (sometimes inverted circadian rhythm), low concentration and poor personal care [5] [9] [14]. Moreover, hikikomori cases feel a pervasive sense of apathy, and a lack of motivation to face life [7]. Researchers indicate that almost 33\% - 36\% of hikikomori have a comorbid psychiatric diagnosis [5], albeit the degree of comorbidity varies depending on study methodology and sampling. A Japanese study, for instance, examined 337 cases of hikikomori divided in three diagnostic subgroups: schizophrenia, mood and anxiety disorders, developmental disorders and personality disorders. In one case only, it was not possible the inclusion in an existing DSM or ICD categories [3].

Some authors suggested a correlation with autism spectrum disorder, schizoid or avoidant personality disorders, cannabis abuse with amotivational syndrome, or even Internet addiction [9]. A recent study identified a group of psychiatric disorders characterised by hikikomori-like features including psychosis, social anxiety disorder, avoidant personality disorder, depressive disorders, Internet addiction and post-traumatic stress disorder [4]. There is also a small but growing body of research on physical conditions of hikikomori and biomarkers that might be associated with this behaviour.

\section{Social Withdrawal}

Social withdrawal is widely accepted as the essential characteristic of hikikomori. 
In a review, $\mathrm{Li}$ et al. [15] tried to analyze the phenomenon of social withdrawal in relation to structural aspects of family and society, as well as behavioural and psychological factors. They suggested three types of social withdrawal processes with different possibilities of intervention and treatment for each: 1) young, over-dependent individuals, who represent the "hard core" pattern of isolation, avoiding all social interactions and secluding themselves at home in their bedrooms, presenting the most severe subjects to reach and treat. These individuals typically grow up in overprotective families and their parents play the role of safe harbour and reinforce secure attachment; 2) maladaptive, interdependent youths who grow up in dysfunctional families and are then unable to learn and build satisfactory relationships. They often drop out of school but occasionally come and go from the seclusion of home; 3) Counter-dependent, socially withdrawn youths who are burdened by the high expectations of their parents. They are expected to spend all their time studying in order to meet the high expectations of their ambitious parents (Table 2). Their socio-economic situation may require them to deal with a lack autonomy and opportunity, contributing to feelings of intense frustration and consequent retirement from social relationships.

Social isolation and loneliness may affect mental and physical health. A previous study on a large American sample found that isolation can lead to major risk of depressive and anxiety disorders, such as major depression, dysthymia, social anxiety and generalized anxiety disorder [36].

A meta-analysis also demonstrated that loneliness is dangerous in term of mortality, as alcohol and smoking abuse [37].

Isolation concurs to several physical and psychiatric dysfunctions that include alcoholism, suicidal thoughts, aggressive behaviours and social anxiety. Loneliness further increases the risk for cognitive impairment, hypertension, increased hypothalamic pituitary adreno-cortical activity, while diminishing immunity and pro-inflammatory gene transcripts [38].

In particular, hikikomori withdrawal may decrease skills in terms of communication and socialization [14].

\section{Internet Addiction and Internet Gaming Disorder}

Although problems with interpersonal relationships and a lack of interest in social activities are common to both hikikomori and those with Internet addiction, the use of Internet by hikikomori should be considered as ego-syntonic [39]. Some authors have highlighted that the use of Internet may be advantageous to hikikomori, since it creates a non-conventional way to interact with others and interrupt the social isolation [23] [40]. At the same time, the widespread of social interactions through online communication may be a contributing factor of social withdrawal [10]. Moreover, hikikomori has been demonstrated to be highly associated with Internet gaming disorder (IGD), and interventions aimed at reducing IGD development in social withdrawal youths have been claimed [16]. 
Despite these potentially negative aspects, the Internet has been also indicated as a useful tool that can provide a more acceptable means of interaction between health professionals and their hikikomori patients [41].

\section{Medical Comorbidity and Biomarkers}

A study from Hong Kong found that hikikomori have a higher incidence of hypertension and pre-hypertension, partially associated with their sedentary lifestyle in combination with unhealthy dietary habits, insufficient physical activities and reversed sleep patterns [42]. However, the results of a one year longitudinal study by the same authors demonstrated that, surprisingly, blood pressure levels of hikikomori were improved by moderate-intensity daily exercises even when all other lifestyle aspects remained the same [43]. Meanwhile, research into blood biomarkers shows that oxidative stress and inflammation may contribute to the behavioural and psychological traits of hikikomori, thus suggesting a biological basis for the condition [44].

\section{Ongoing Issues with Hikikomori Management}

The first contact with healthcare usually occurs long after hikikomori behaviours are established, with care delayed as a result of both the social withdrawal itself and possible resistance by the family, whose sense of guilt, shame, stigmatization, or lack of knowledge may result in a delayed consultation request [10]. For these reasons, the greatest barrier identified for treatment of hikikomori is the failure to reach the healthcare setting [6] [12] [14] [15] [19] [45].

Another treatment issue involves the differentiation between primary and secondary hikikomori, since the former requires specific interventions while interventions for the latter should primarily address the comorbid mental disorder [10]. Cultural differences in the causes and interpretation of social withdrawal should be accounted for in its management. For example, in Asia, withdrawal from society is a way of escaping group conformity and mainstream culture, while in Western countries withdrawal may be a reaction to individual difficulties [41].

When it comes to interventions addressed to hikikomori, the literature is concentrated mainly on Japan [6] [10] [11], although specific interventions have also been described in other countries, such as Spain [20], South Korea [14], and Italy [8]. Japan is the only country where targeted services are relatively widespread and systematically organized, including healthcare settings specifically dedicated to hidden youth withdrawal [10] [41]. Three main types of health care services are provided in Japan:

1) Mental care facilities using clinical support and psycho-social support with individual or group counseling;

2) Community services using non-clinical approaches with the aim of re-socializing hikikomori through different methods, such as group participation and communication skills training; and 
3) Alternative treatments, such as pet-therapy, communal cooking on farms or online platforms.

In general, the management of hikikomori should follow an integrated approach. Once identified, an initial assessment including a comprehensive clinical evaluation can determine the presence of comorbid mental disorders. Should comorbidities be present, the therapeutic approach generally used for the specific psychiatric disorder should be applied. This may include appropriate pharmacological treatments and even hospitalization when severe symptoms are present. A study from Spain confirmed rapid and stable improvement over one year in hikikomori who underwent intensive treatment such as hospitalization [20]. In contrast, the psychiatric treatment alone has been demonstrated to be potentially harmful in hikikomori patients [11].

The web, however, may be a useful channel for interpersonal communication at home. A study found that socially withdrawn youths use the Internet as a platform for communication. Online counseling services through Facebook, WhatsApp, or Twitter, can offer secure and distanced channel for socially withdrawn youths to reestablish their trust in others, before face-to-face interventions can be initiated [14].

Literature reports few experiences of internet-based therapeutic approaches for hikikomori. Games and communication instruments using computers and smartphones are rapidly developing, and emerging technologies may help some groups of hikikomori [28].

For instance, an online game tools called "Pokemon Go", released in 2016 and using location information and augmented reality, initially seemed to be helpful in supporting hikikomori people. In fact, people who had been locked in their house for years began to go out to look for "Pokemons". Unfortunately this effect was only transient, but this does not exclude that an internet-based approach could be supportive in the early stage of the isolation [46] [47]. In contrast to their expectations, however, Kato et al. found that quite a number of hikikomori people express the desire for a direct face-to-face treatment [4].

When primary hikikomori is diagnosed, mainly psychological and psychotherapeutic interventions are to be used. These can include counselling services, home visitation programs with brief therapeutic interventions, family or group activities, and support groups [6] [10] [14] [15] [45]. Other interventions can include psychodynamic therapy or nidotherapy, as well as attempts to alter the physical and social environment to help create a better fit for hikikomori [10] [23] [48]. The literature generally indicates that treatment of hikikomori can be long and sometimes non-performing since it can be difficult to convince the patient to engage in the therapeutic process and response rates are very low [10] [23] [49]. Notably, there is no evidence that pharmacological treatments are effective.

Research to date suggests that primary hikikomori may benefit mainly from psychosocial treatment, although clinical evaluation should be scheduled as a 
follow-up measure to guard against the possible onset of psychiatric symptoms. When social reintegration into society is achieved, hikikomori should be further supported since social withdrawal can pose further serious issues in terms of years of school or work lost. While the severity of the phenomenon varies on an individual basis, in the most extreme cases people may remain isolated for years or even decades [50]. In Japan, many thousands of people have been reported as a hikikomori individual for longer than twenty years [50]; this means that they are, totally unable to live normally. In the generational transition, at the death of the parents, they will represent a very relevant social problem.

Clinical help and family support are deemed to be critical to recovery; there is evidence that the prognosis can be worse when either or both are absent [10] [12].

To date, the international literature seems to pay increasing attention to the phenomenon of hikikomori, albeit there is not yet a clear indication of the global prevalence of this phenomenon [30]. As highlighted, the intervention of healthcare professionals may often be required for these people, especially in case of psychiatric comorbidity. However, severe social withdrawal, which is one of the main aspects of hikikomori phenomenon, may be seen as an alternative coping strategy, or the choice of belonging to a subculture, which makes the individual feel integrated when there is no recognition or placement within the common social system. In any case-as reported in this article-several types of treatment approaches can be effective for these people.

\section{Conflicts of Interest}

The authors declare no conflicts of interest regarding the publication of this paper.

\section{References}

[1] Furuhashi, T., Tsuda, H., Ogawa, T., Suzuki, K., Shimizu, M., Teruyama, J., et al. (2013) Current Situation, Commonalities and Differences between Socially Withdrawn Young Adults (Hikikomori) in France and Japan. Evolution Psychiatrique, 78, 249-266. https://doi.org/10.1016/j.evopsy.2013.01.016

[2] Kaneko, S. (2006) Japan's “Socially Withdrawn Youths” and Time Constraints in Japanese Society-Management and Conceptualization of Time in a Support Group for "Hikikomori". Time \& Society, 15, 233-249. https://doi.org/10.1177/0961463X06067034

[3] Tajan, N. (2015) Social Withdrawal and Psychiatry: A Comprehensive Review of Hikikomori. Neuropsychiatrie de I Enfance et de I Adolescence, 63, 324-331. https://doi.org/10.1016/j.neurenf.2015.03.008

[4] Kato, T.A., Kanba, S. and Teo, A.R. (2019) Hikikomori: Multidimensional Understanding, Assessment, and Future International Perspectives. Psychiatry and Clinical Neurosciences, 73, 427-440. https://doi.org/10.1111/pcn.12895

[5] Aguglia, E., Signorelli, M.S., Pollicino, C., Arcidiacono, E. and Petralia, A. (2010) Hikikomori Phenomenon: Cultural Bound or Emergent Psychopathology? Giorn Ital Psicopat, 16, 157-164. 
[6] Teo, A.R. (2010) A New Form of Social Withdrawal in Japan: A Review of Hikikomori. International Journal of Social Psychiatry, 56, 178-185. https://doi.org/10.1177/0020764008100629

[7] Furlong, A. (2008) The Japanese Hikikomori Phenomenon: Acute Social Withdrawal among Young People. Sociological Review, 56, 309-325.

https://doi.org/10.1111/j.1467-954X.2008.00790.x

[8] Ranieri, F. and Luccherino, L. (2018) Hikikomori: Debating a XXI Century Phenomenon from a Clinical Point of View. Scandinavian Journal of Child and Adolescent Psychiatry and Psychology, 6, 72-79. https://doi.org/10.21307/sjcapp-2018-002

[9] Kato, T.A., Tateno, M., Shinfuku, N., Fujisawa, D., Teo, A.R., Sartorius, N., et al. (2012) Does the "Hikikomori" Syndrome of Social Withdrawal Exist outside Japan? A Preliminary International Investigation. Social Psychiatry and Psychiatric Epidemiology, 47, 1061-1075. https://doi.org/10.1007/s00127-011-0411-7

[10] Stip, E., Thibault, A., Beauchamp-Chatel, A. and Kisely, S. (2016) Internet Addiction, Hikikomori Syndrome, and the Prodromal Phase of Psychosis. Frontiers in Psychiatry, 7, Article No. 6. https://doi.org/10.3389/fpsyt.2016.00006

[11] Yong, R. and Nomura, K. (2019) Hikikomori Is Most Associated with Interpersonal Relationships, Followed by Suicide Risks: A Secondary Analysis of a National CrossSectional Study. Frontiers in Psychiatry, 10, 247.

https://doi.org/10.3389/fpsyt.2019.00247

[12] Malagon-Amor, A., Corcoles-Martinez, D., Martin-Lopez, L.M. and Perez-Sola, V. (2015) Hikikomori in Spain: A Descriptive Study. International Journal of Social Psychiatry, 61, 475-483. https://doi.org/10.1177/0020764014553003

[13] Sakamoto, N., Martin, R.G., Kumano, H., Kuboki, T. and Al-Adawi, S. (2005) Hikikomori, Is It a Culture-Reactive or Culture-Bound Syndrome? Nidotherapy and a Clinical Vignette from Oman. International Journal of Psychiatry in Medicine, 35, 191-198. https://doi.org/10.2190/7WEQ-216D-TVNH-PQJ1

[14] Lee, Y.S., Lee, J.Y., Choi, T.Y. and Choi, J.T. (2013) Home Visitation Program for Detecting, Evaluating and Treating Socially Withdrawn Youth in Korea. Psychiatry and Clinical Neurosciences, 67, 193-202. https://doi.org/10.1111/pcn.12043

[15] Li, T.M.H. and Wong, P.W.C. (2015) Youth Social Withdrawal Behavior (Hikikomori): A Systematic Review of Qualitative and Quantitative Studies. Australian and New Zealand Journal of Psychiatry, 49, 595-609. https://doi.org/10.1177/0004867415581179

[16] Stavropoulos, V., Anderson, E.E., Beard, C., Latifi, M.Q., Kuss, D. and Griffiths, M. (2019) A Preliminary Cross-Cultural Study of Hikikomori and Internet Gaming Disorder: The Moderating Effects of Game-Playing Time and Living with Parents. Addictive Behaviors Reports, 9, Article ID: 100137. https://doi.org/10.1016/j.abrep.2018.10.001

[17] Kato, T.A., Kanba, S. and Teo, A.R. (2018) Hikikomori: Experience in Japan and International Relevance. World Psychiatry, 17, 105-106. https://doi.org/10.1002/wps.20497

[18] Suwa, M. and Suzuki, K. (2013) The Phenomenon of "Hikikomori" (Social Withdrawal) and the Socio-Cultural Situation in Japan Today. Journal of Psychopathology, 19, 191-198.

[19] Koyama, A., Miyake, Y., Kawakami, N., Tsuchiya, M., Tachimori, H., Takeshima, T., et al. (2010) Lifetime Prevalence, Psychiatric Comorbidity and Demographic Correlates of "Hikikomori" in a Community Population in Japan. Psychiatry Research, 176, 69-74. https://doi.org/10.1016/j.psychres.2008.10.019 
[20] Malagon-Amor, A., Martin-Lopez, L.M., Corcoles, D., Gonzalez, A., Bellsola, M., Teo, A.R., et al. (2018) A 12-Month Study of the Hikikomori Syndrome of Social Withdrawal: Clinical Characterization and Different Subtypes Proposal. Psychiatry Research, 270, 1039-1046. https://doi.org/10.1016/j.psychres.2018.03.060

[21] Sugai, M. (2016) Hikikomori; An Investigation into the Socio-Cultural and Socio-Economic Constructs Influencing Specific Symptomatology of Hikikomori in Japan. The Forum, 30, 15-26.

[22] Teo, A.R. and Gaw, A.C. (2010) Hikikomori, a Japanese Culture-Bound Syndrome of Social Withdrawal? A Proposal for DSM-5. Journal of Nervous and Mental Disease, 198, 444-449. https://doi.org/10.1097/NMD.0b013e3181e086b1

[23] Teo, A.R., Fetters, M.D., Stufflebam, K., Tateno, M., Balhara, Y., Choi, T.Y., et al. (2015) Identification of the Hikikomori Syndrome of Social Withdrawal: Psychosocial Features and Treatment Preferences in Four Countries. International Journal of Social Psychiatry, 61, 64-72. https://doi.org/10.1177/0020764014535758

[24] Sakai, M., Ishikawa, S.-I., Sato, H. and Sakano, Y. (2004) Development of Hikikomori Behavior Checklist (HBCL) and Examination of Its Reliability and Validity. Japanese Journal of Counselling Science, 37, 210-220.

https://doi.org/10.1037/t68812-000

[25] Nonaka, S., Shimada, H. and Sakai, M. (2018) Assessing Adaptive Behaviors of Individuals with Hikikomori (Prolonged Social Withdrawal, Development and Psychometric Evaluation of the Parent-Report Scale). International Journal of Culture and Mental Health, 11, 280-294. https://doi.org/10.1080/17542863.2017.1367411

[26] Uchida, Y. and Norasakkunkit, V. (2015) The NEET and Hikikomori Spectrum: Assessing the Risks and Consequences of Becoming Culturally Marginalized. Frontiers in Psychology, 6, 1117. https://doi.org/10.3389/fpsyg.2015.01117

[27] Teo, A.R., Chen, J.I., Kubo, H., Katsuki, R., Sato-Kasai, M., Shimokawa, N., et al. (2018) Development and Validation of the 25-Item Hikikomori Questionnaire (HQ-25). Psychiatry and Clinical Neurosciences, 72, 780-788. https://doi.org/10.1111/pcn.12691

[28] Kato, T.A., Shinfuku, N. and Tateno, M. (2020) Internet Society, Internet Addiction, and Pathological Social Withdrawal: The Chicken and Egg Dilemma for Internet Addiction and Hikikomori. Current Opinion in Psychiatry, 33, 264-270. https://doi.org/10.1097/YCO.0000000000000601

[29] Tajan, N., Yukiko, H. and Pionnie-Dax, N. (2017) Hikikomori: The Japanese Cabinet Office's 2016 Survey of Acute Social Withdrawal. Asia-Pacific Journal-Japan Focus, 15,1 .

[30] Pozza, A., Coluccia, A., Kato, T., Gaetani, M. and Ferretti, F. (2019) The "Hikikomori" Syndrome: Worldwide Prevalence and Co-Occurring Major Psychiatric Disorders: A Systematic Review and Meta-Analysis Protocol. BMJ Open, 9, e025213. https://doi.org/10.1136/bmjopen-2018-025213

[31] Wong, P.W.C., Li, T.M.H., Chan, M., Law, Y.W., Chau, M., Cheng, C., et al. (2015) The Prevalence and Correlates of Severe Social Withdrawal (Hikikomori) in Hong Kong: A Cross-Sectional Telephone-Based Survey Study. International Journal of Social Psychiatry, 61, 330-342. https://doi.org/10.1177/0020764014543711

[32] Norasakkunkit, V. and Uchida, Y. (2014) To Conform or to Maintain Self-Consistency? Hikikomori Risk in Japan and the Deviation from Seeking Harmony. Journal of Social and Clinical Psychology, 33, 918-935. https://doi.org/10.1521/jscp.2014.33.10.918

[33] Zielenziger, M. (2006) Shutting Out the Sun: How Japan Created Its Own Lost 
Generation. Nan A. Talese, New York.

[34] Funakoshi, A. and Miyamoto, Y. (2015) Significant Factors in Family Difficulties for Fathers and Mothers Who Use Support Services for Children with Hikikomori. Psychiatry and Clinical Neurosciences, 69, 210-219. https://doi.org/10.1111/pcn.12230

[35] Tateno, M., Park, T.W., Kato, T.A., Umene-Nakano, W. and Saito, T. (2012) Hikikomori as a Possible Clinical Term in Psychiatry: A Questionnaire Survey. BMC Psychiatry, 12, 169. https://doi.org/10.1186/1471-244X-12-169

[36] Teo, A.R. (2013) Social Isolation Associated with Depression: A Case Report of Hikikomori. International Journal of Social Psychiatry, 59, 339-341.

https://doi.org/10.1177/0020764012437128

[37] van Beljouw, I.M.J., Verhaak, P.F.M., Cuijpers, P., van Marwijk, H.W.J. and Penninx, B. (2010) The Course of Untreated Anxiety and Depression, and Determinants of Poor One-Year Outcome: A One-Year Cohort Study. BMC Psychiatry, 10, 86. https://doi.org/10.1186/1471-244X-10-86

[38] Cacioppo, S., Grippo, A.J., London, S., Goossens, L. and Cacioppo, J.T. (2015) Loneliness: Clinical Import and Interventions. Perspectives on Psychological Science, 10, 238-249. https://doi.org/10.1177/1745691615570616

[39] Cerniglia, L., Zoratto, F., Cimino, S., Laviola, G., Ammaniti, M. and Adriani, W. (2017) Internet Addiction in Adolescence: Neurobiological, Psychosocial and Clinical Issues. Neuroscience and Biobehavioral Reviews, 76, 174-184.

https://doi.org/10.1016/j.neubiorev.2016.12.024

[40] Taylor, M. (2006) Strategies of Dissociation: A Mimetic Dimension to Social Problems in Japan. Anthropoetics, 12, 11.

[41] Chan, G.H.Y. and Lo, T.W. (2014) Hidden Youth Services: What Hong Kong Can Learn from Japan. Children and Youth Services Review, 42, 118-126.

https://doi.org/10.1016/j.childyouth.2014.03.021

[42] Yuen, J.W.M., Yan, Y.K.Y., Wong, V.C.W., Tam, W.W.S., So, K.W. and Chien, W.T. (2018) A Physical Health Profile of Youths Living with a "Hikikomori" Lifestyle. International Journal of Environmental Research and Public Health, 15, 315. https://doi.org/10.3390/ijerph15020315

[43] Yuen, J.W.M., Wong, V.C.W., Tam, W.W.S., So, K.W. and Chien, W.T. (2019) A One-Year Prospective Follow-Up Study on the Health Profile of Hikikomori Living in Hong Kong. International Journal of Environmental Research and Public Health, 16, 546. https://doi.org/10.3390/ijerph16040546

[44] Hayakawa, K., Kato, T.A., Watabe, M., Teo, A.R., Horikawa, H., Kuwano, N., et al. (2018) Blood Biomarkers of Hikikomori, a Severe Social Withdrawal Syndrome. Scientific Reports, 8, Article No. 2884. https://doi.org/10.1038/s41598-018-21260-w

[45] Kondo, N., Sakai, M., Kuroda, Y., Kiyota, Y., Kitabata, Y. and Kurosawa, M. (2013) General Condition of Hikikomori (Prolonged Social Withdrawal) in Japan: Psychiatric Diagnosis and Outcome in Mental Health Welfare Centres. International Journal of Social Psychiatry, 59, 79-86. https://doi.org/10.1177/0020764011423611

[46] Kato, T.A., Teo, A.R., Tateno, M., Watabe, M., Kubo, H. and Kanba, S. (2017) Can Pokémon GO Rescue Shut-Ins (Hikikomori) from Their Isolated World? Psychiatry and Clinical Neurosciences, 71, 75-76. https://doi.org/10.1111/pcn.12481

[47] Tateno, M., Skokauskas, N., Kato, T.A., Teo, A.R. and Guerrero, A.P.S. (2016) New Game Software (Pokémon Go) May Help Youth with Severe Social Withdrawal, Hikikomori. Psychiatry Research, 246, 848-849. https://doi.org/10.1016/j.psychres.2016.10.038 
[48] Wilson, S. (2010) Braindance of the Hikikomori: Towards a Return to Speculative Psychoanalysis. Paragraph, 33, 392-409. https://doi.org/10.3366/para.2010.0206

[49] Nagata, T., Yamada, H., Teo, A.R., Yoshimura, C., Nakajima, T. and van Vliet, I. (2013) Comorbid Social Withdrawal (Hikikomori) in Outpatients with Social Anxiety Disorder: Clinical Characteristics and Treatment Response in a Case Series. International Journal of Social Psychiatry, 59, 73-78.

https://doi.org/10.1177/0020764011423184

[50] Ricci, C. (2008) Hikikomori: Adolescenti in volontaria reclusione [Hikikomori: Adolescents in Voluntary Seclusion]. Angeli F, Milano. 